with nedocromil sodium and placebo there were only two treatment differences in favour of nedocromil sodium: the rate of withdrawals, all due to exacerbations, was significantly lower during treatment with nedocromil sodium, whereas clinician opinion of treatment efficacy significantly favoured nedocromil sodium.

There are several explanations why treatment with nedocromil sodium in our patients with COPD failed to improve clinical parameters. It is possible that nedocromil sodium is not a potent anti-inflammatory agent in this patient group; however, if nedocromil sodium is effective the duration of treatment may have been too short, as has been suggested for other anti-inflammatory agents. ${ }^{4}$ The type of airway inflammation, which is different in asthmatics ${ }^{5}$ who generally respond better to anti-inflammatory medication, ${ }^{6}$ may also play a part. Instead of symptom scores, lung function, and airway responsiveness, other clinical parameters such as quality of life, decline in pulmonary function, or number and duration of exacerbations may be necessary to measure treatment efficacy in COPD. ${ }^{7}$ In this perspective our patients showed, in the short period of follow up, significantly fewer exacerbations when treated with nedocromil sodium. However, at the expense of treatment with nedocromil sodium, the higher withdrawal rate in the placebo group leads to selection bias, thus underestimating differences between placebo and nedocromil sodium treatment.
In conclusion, 10 weeks of treatment with a high daily dosage of nedocromil sodium did not improve pulmonary function, airway responsiveness, and clinical symptoms. The number of withdrawals, all due to exacerbations, was significantly greater in the placebo group than in the nedocromil sodium group. Longer trials will be necessary to determine whether nedocromil sodium reduces the frequency of exacerbations and the decrease in pulmonary function, eventually leading to a better quality of life in patients with COPD.

This work was supported by a grant from Fisons Plc, Pharmaceutical Division.

1 de Jong JW, Postma DS, de Monchy JGR, Koëter GH. A review of nedocromil sodium in asthma therapy. Eur Respir Rev 1993;15:511-9.

2 ATS. Standards for the diagnosis and care of patients with chronic obstructive pulmonary disease. Am Rev Respir Dis 1987;130:225-44.

3 Oosterhoff Y, de Jong JW, Jansen MAM, Koëter GH, Postma DS. Airway responsiveness to adenosine $5^{\prime}$-monophosphate in chronic obstructive pulmonary disease is determined by smoking. Am Rev Respir Dis 1993;147:553-8.

4 Auffarth B, Postma DS, de Monchy JGR, van der Mark ThW, Boorsma M, Koëter GH. Effects of inhaled budesonide on spirometric values, reversibility, airway responsiveness, and cough threshold in smokers with chronic obstructive lung disease. Thorax 1991;46:372-7.

5 Ollerenshaw SL, Woolcock AJ. Characteristics of the inflammation in biopsies from large airways of subjects with asthma and subjects with chronic airflow limitation. $\mathrm{Am}$ asthma and subjects with chronic
Rev Respir Dis 1992;145:922-7.

6 Juniper EF, Kline PA, Vanzieleghem MA, Ramsdale EH, O'Byrne PM, Hargreave FE. Long-term effects of budesonide on airway responsiveness and clinical asthma severity in inhaled steroid-dependent asthmatics. Eur Respir f 1990;3:1122-7.

7 Callahan CM, Dittus RS, Katz BP. Oral corticosteroid therapy for patients with stable chronic obstructive pulmonary disease. Ann Intern Med 1991;114:216-23.

\section{Effect of inhaled menthol on citric acid induced cough in normal subjects}

\author{
A H Morice, A E Marshall, K S Higgins, T J Grattan
}

Conclusions - Menthol is an effective antitussive agent in an evoked cough model.

(Thorax 1994;49:1024-1026)

University
Department of
Medicine and
Pharmacology, Royal
Hallamshire Hospital,
Sheffield S10 2JF, UK
A H Morice
A E Marshall
K S Higgins
T J Grattan
Reprint requests to:
Dr A H Morice.
Received 9 February 1994
Returned to authors
5 April 1994
Revised version received
7 June 1994
Accepted for publication
9 June 1994

\begin{abstract}
Background - Menthol is a commonly used ingredient in many over the counter cough remedies, but there is little objective evidence as to its efficacy.

Methods - Twenty healthy subjects received a cough challenge consisting of five inhalations of $33 \mu \mathrm{mol}$ citric acid from an air driven dosimeter. The challenge was repeated at hourly intervals for five hours. Five minutes before each challenge subjects inhaled, in a randomised design, either menthol $75 \%$ in eucalyptus oil or one of two placebos (pine oil or air).

Results - Menthol inhalation caused a reduction in evoked cough when compared with either placebo.
\end{abstract}

L-Menthol, a volatile aromatic compound, is the principal component of the essential oil derived from peppermint (Mentha piperita). L-Menthol and the synthetic racemate DLmenthol are included in a number of proprietary medicines sold for the treatment of symptoms due to the common cold.

In this study we have investigated the effect of menthol inhalation in a citric acid induced cough model and compared its effects with that of two placebo treatments, the first an inhaler 


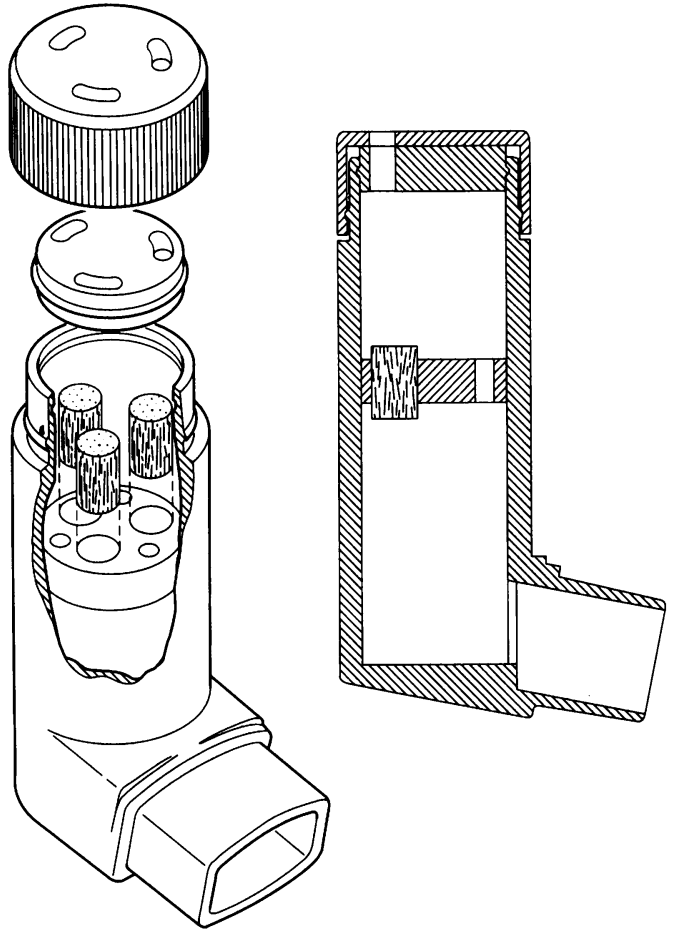

Figure 1 Diagram of the SmithKline Beecham inhalation device for aromatic vapours.

device containing no medication and the second an inhaler containing pine oil dissolved in liquid paraffin. Pine oil was chosen as the second placebo as it was considered that the medicinal aroma would assist in the blinding of the study. It has previously been shown to be without antitussive activity. ${ }^{1}$

\section{Methods}

Twenty normal subjects (13 women; mean age 24 (range 20-46) years) free from recent re-

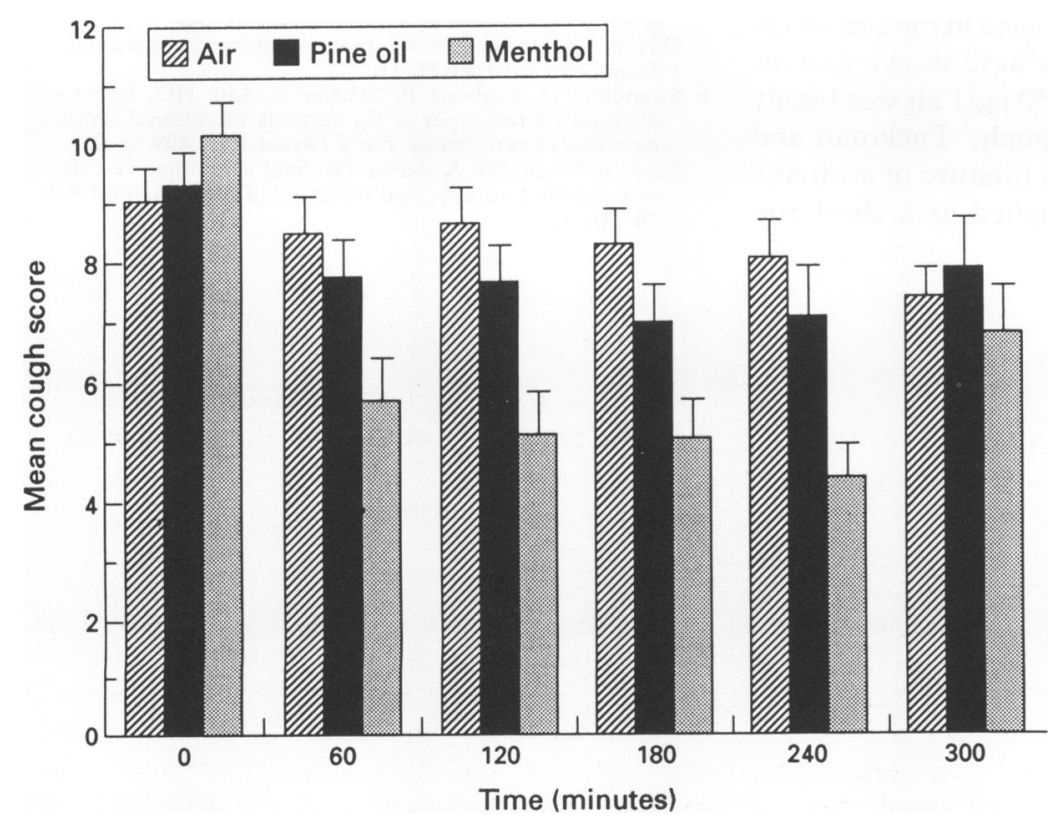

Figure 2 Mean number of coughs following treatment with air, pine oil, and menthol. Significant differences in cough count between menthol and pine oil treatments were seen at one hour $(p<0.0005)$, two hours $(p<0.0001)$, three hours $(p<0.002)$, four hours $(p<0.0002)$, and five hours $(p<0.05)$. Significant differences between menthol and air treatments were seen at one, two, three, and four hours $(p<0 \cdot 0001)$, but at five hours there was no significant difference. spiratory tract infection were studied using an inhalation cough challenge. Citric acid was delivered by a compressed air driven nebuliser controlled by a breath activated dosimeter (Mefar, Brescia, Italy). Five single inhalations were administered and the dosimeter was preset to limit the delivery time to one second, with a 60 second interval between each of the five inhalations. The mean nebuliser output was $0.125 \mathrm{ml}$ per inhalation and the dose delivered was approximately $33 \mu \mathrm{mol}$ citric acid. The cough response in the 60 seconds following each inhalation was recorded by an observer. Individuals were screened on two occasions and only those subjects with a reproducible cough response of between five and 15 coughs were accepted into the study protocol.

Subjects were asked to return to the clinic on three occasions at the same time of day, having fasted from midnight. Each study day was separated by at least 24 hours and on each occasion a baseline cough challenge was performed to ensure that cough sensitivity had not altered between study days. Fifty five minutes after the baseline cough challenge subjects received, in a randomised double blind protocol, the study medication consisting of pine oil, air, or menthol. The menthol treatment also contained eucalyptus oil, the purpose of which was to solubilise the menthol and also to enhance the organoleptic properties of the product. The study was approved by the local ethical committee.

\section{MATERIALS}

Inhalation device

Each treatment was administered via the SmithKline Beecham inhalation device depicted in fig 1. Each of the larger channels contained a cylindrical wick $(7 \mathrm{~mm} \times 22 \mathrm{~mm})$ made from cellulose acetate fibre which was impregnated with the materials under test. The smaller channels were unfilled and served to allow the passage of a quantity of unaromatised air such that the final concentration, as estimated by gas chromatography at a flow rate of $6 \mathrm{l} / \mathrm{min}$, was approximately $20 \mu \mathrm{g} / \mathrm{l}$ for menthol and $200 \mu \mathrm{g} / 1$ for cineole (the principal component of eucalyptus oil).

\section{Test formulations}

The materials impregnated onto the wicks were (1) menthol $75 \%$ w/w (Harman and Reimer, Holzminden, Germany) dissolved in eucalyptus oil $25 \% \mathrm{w} / \mathrm{w}(0.5 \mathrm{~g} /$ wick) (Bushboake Allen, London, UK); (2) pine oil (sylvestris) $0.5 \% \mathrm{w} / \mathrm{w}$ (William Ransom, Hichen, UK) dissolved in liquid paraffin $99.5 \% \mathrm{w} / \mathrm{w}$ ( $0.5 \mathrm{~g} /$ wick) (Karless Refining, Romford, UK); or (3) none.

Cough challenge was repeated at 60,120 , 180,240 , and 300 minutes after the initial challenge. Five minutes before each challenge except the last - that is, at $55,115,175$, and 235 minutes after the initial cough challenge subjects were administered inhalations of the test vapours over five minutes from the inhalation device. 
STATISTICAL ANALYSIS

The two primary end points to be analysed were the reduction in the number of coughs after dosing compared with the number of coughs at the two non-treatment time points (the first and last cough challenge), and the area under the cough response curve - that is, the total number of coughs on each day. The results of each treatment were compared by analysis of variance.

\section{Results}

Mean cough per challenge is shown in fig 2 . Treatment with pine oil and air resulted in no significant reduction in cough frequency during the course of the experiment. Menthol produced a reduction in cough frequency at all time points which was highly significant $(p<0.0005)$ with a mean (SE) reduction of cough frequency to 1734 (169) compared with 2293 (174) for pine oil and 2509 (158) for air. At the end of the study, when subjects had not received any medication for 65 minutes before challenge, cough was still significantly reduced $(p<0.05)$, but only by $10 \%$ when compared with placebo treatment.

\section{Discussion}

In this study repeated administration of menthol was used because it is highly volatile and therefore rapidly eliminated during exhalation. This regimen mimics that which occurs in patients taking repeated doses of over the counter medicines. We have found menthol to be a highly effective antitussive agent when taken by this method. The citric acid cough challenge model has been widely used and is highly reproducible provided that the short term tachyphylaxis seen with citric acid is allowed for in the study design. ${ }^{2}$

The efficacy of menthol as an antitussive agent is supported by previous studies carried out in our laboratory using a citric acid in the conscious guinea pig model where we have shown that inhaled menthol at levels of $30 \mu \mathrm{g} / 1$ air was highly effective in suppressing cough. ${ }^{3}$ Packman and London have shown that a mixture of aromatic oils including menthol applied as a chest rub to humans produces a significant reduction in citric acid induced cough 30 and 60 minutes after administration. ${ }^{1}$

Unlike other common cough treatments such as dextromethorphan and codeine, which are thought to act centrally, the mechanism of the antitussive action of menthol is probably localised to the airways. The cooling sensation which is felt with administration of menthol has been ascribed to stimulation of cold receptor nerve endings. ${ }^{4}$ This may be analogous to the hot sensation generated by the pungent extract of red peppers (capsaicin). Capsaicin has been found to alter sodium flux through small afferent $C$ fibre neurones by binding to specific cell membrane receptors. ${ }^{5}$ These neurones then depolarise leading to generation of action potentials and the perception of irritation which is expressed in the oropharynx as a hot sensation and in the airways as cough. Whether menthol has a similar specific site of action is at present unclear, but it has been postulated that menthol exerts its effects by interfering with the calcium conductance across the sensory neuronal membrane. ${ }^{6}$ In the airways menthol has been shown to stimulate laryngeal cold receptors in the absence of cold air with no effect on laryngeal mechanoreceptors. ${ }^{7}$ It is an interesting observation that two compounds which alter sensory perception in opposite directions in the oropharynx have similar opposite activity on the cough reflex.

The authors wish to thank SmithKline Beecham Ltd who supplied the inhalation device.

1 Packman EW, London SJ. The utility of artificially induced cough as a clinical model for evaluating the antitussive effects of aromatics delivered by inunction. Eur Respir $\mathcal{f}$ 1980;61:101-9.

2 Morice AH, Higgins KS, Yeo WW. Adaptation of cough reflex with different types of stimulation. Eur Respir 7 1992; 5:841-7.

3 Laude EA, Grattan TJ, Morice AH. The effects of menthol and aromatic vapours on citric acid induced cough in guinea pigs. Pulm Pharmacol 1994;(in press)

4 Hensel H, Zotterman Y. The effect of menthol on the thermal receptors. Acta Physiol Scand 1951;24:27-34.

5 O'Neill TP. Mechanism of capsaicin action; recent learnings. Respir Med 1991;85:35-41.

6 Swandulla D, Carbone E, Schafer K, Lux HD. Effect of menthol on two types of $\mathrm{Ca}$ currents in cultured sensory neurones of vertebrates. Eur f Physiol 1987;409:52-9.

7 Sant' Ambrogio FB, Anderson JW, Sant' Ambrogio G. Effect of L-menthol on laryngeal receptors. F Appl Physiol 1991; 70:788-93. 